\title{
A Multimedia Data Mining Framework For MONITORING E-EXAMINATION ENVIRONMENT
}

\author{
Adetoba, B. T., Awodele, O. and Kuyoro, S. O. \\ Department of Computer Science, Babcock University, Ilishan, Ogun State
}

\begin{abstract}
Academic dishonesty has been a growing concern in e-learning environment due to the fact that $e$ examination takes place under supervised and unsupervised learning environment despite its huge advantages. The e-examination environment has faced various security breaches such as academic dishonesty (impersonation), identity theft, unauthorised access and illegal assistance as a result of inefficient measures employed. Hence, an efficient framework which will aid the monitoring of the $e$ examination is needed. This paper reviews the process of mining multimedia data and propose a framework for monitoring the e-examination environment in order to extract images and audio features. The framework has four major phases: data pre-processing, mining, association and post processing. The pre-processing phases carries out the extraction and transformation of multimedia data features, the mining phase does the classification and clustering of these features, the association does pattern matching while the post processing carries out the knowledge interpretation and reporting. The approach presented in this study will allow for efficient and accurate monitoring of e-examination environment which will help provide adequate security and reduce unethical behaviour in e-examination environment.
\end{abstract}

\section{KEYWORDS}

Data mining, E-examination, E-examination environment, Multimedia Data, Security

\section{INTRODUCTION}

Student assessment in an e-learning environment has not been without challenges. Studies have shown that academic dishonesty is prominent at all levels of education in developing and developed countries [1]. Many studies reported that cheating is common in education. Studies have also been conducted showing the rate of cheating in an online learning environment compare to the traditional face to face environment $[2,3,4]$. Result from some of these studies indicates that cheating occurs more in online test or examination compare to the traditional face to face system i.e. Students receive help or assistance during online quiz/examination [5]. One crucial problem existing in an e-learning platform is the issue of security. Also, there is the inefficiency of the method employed in monitoring e-examination environment which have not been able to properly detect individuals with the same identical facial structure e.g. identical twins. For instance, if only the image is considered, it implies that the two individuals are seen as one by the monitoring system. Also, the problem of integrity especially in an unsupervised environment that occur as a result of weak identity control measure [6].

Past researches have also shown that employing biometrics features provides the most effective and accurate identification method that cannot be stolen or shared. Also, several studies have been conducted showing that using a single biometric alone for monitoring an environment is insufficient. Multiple biometrics combines many human traits such as fingerprint, image or face, eye, voice etc. to identify an unknown individual. Multimedia Data Mining (MDM) is a subfield of data mining which is used in extracting interesting information from multimedia data sets and combination of several types of data set which are all converted from different formats into digital 
media [7, 8]. Multimedia data are either semi structured or unstructured and needs to be converted to structured data. These data are stored in multimedia database. One of the most promising areas of MDM is in biometric for identity verification and monitoring the e-examination environment. Multimodal mining differs in many aspects from single modality mining in the ways it combines different features or in generating more semantically meaningful features.

This paper review the process of mining multimedia data and propose a framework that can be used in monitoring participants in an e-examination environment. This study is not meant to discuss the design or technical issues of the multimedia data mining but to discuss how the concept can be applied to e-examination environment.

\section{RELATED WORKS}

Several research works have been conducted in the area of multimedia data mining by various researchers which have been employed in several domains. So also, some research works have been carried out in e-assessment domain that has to do with the ensuring of integrity. The use of MDM for monitoring e-assessment environment or maintaining integrity is yet to be delved in this area of research.

Amigud et al. [9] proposed an integrated approach to enhance academic integrity of eassessments which was based on biometrics and machine learning. Naive Bayes classifier and word n-gram based feature set were employed in presenting a preliminary result. The approach was based on modeling and not observation. This approach was able to provide continuous identity and assurance of authorship throughout the learning activities. It was also able to measure the collaboration of learners within peer content. The work was limited in that no performance baseline was established and it was not field tested.

Vijayarani and Sikila [8] provided the basic concepts of multimedia mining and its essential characteristics as well as its architectures for structures and unstructured data. They also looked at the research issues that take place in the field of research. This aided providing knowledge in multimedia mining research. It also gave in-depth discussion on the application of multimedia data mining. The work only did not discuss the usage of multimedia data mining for monitoring environments. There was also no practical case study in the discussion.

Pesare et al. [10] designed learning dashboards for the representation of the most interesting learning analytics with the aim of providing teachers and learners with understandable view of learning data which was easy in a virtual learning environment. The approach provided clear vision for student to know their learning gap. Also, the analysis did not require an online access. The e-assessment aspect was not considered in the work and the use of multimedia data for monitoring was neglected.

Dhakar and Tiwani [11] proposed a novel hybrid model for intrusion detection which utilizes data mining classification algorithms. The framework was effective and adaptive. Also, the accuracy of the system showed an effective detection rate and less false alarm rate. The framework was not just based on multimedia data but instead on network communication.

Banda et al. [12] introduced a framework which they called Image FARMER which allowed the retrieval of information for developing and customizing domain-specific content based image data by employing data mining concept. The framework proved to have fast and efficient querying and easier for retrieving images. The deficiency in the work was its sole concentration on image and that it was not implemented in an e-assessment environment. 
Chen et. al. [13] developed a multimedia data mining framework for mining information from traffic video sequences. The purpose was to discover hidden knowledge such as vehicle identification, traffic flow, queue detection, incident detection, and the spatio-temporal relations of the vehicles at intersections which provide an economic approach for daily traffic monitoring operations. The proposed MDM framework analyzes the traffic video sequences using background subtraction, image/video segmentation, vehicle tracking, and modeling with the multimedia augmented transition network (MATN) model and multimedia input strings, in the domain of traffic monitoring over traffic intersections. The framework proved to be capable for traffic video monitoring and was able to provide useful information such as queue detection, vehicle classification, traffic flow, and incident detection at the intersections. The deficiency in this work is that it was not implemented in an e-assessment environment.

The research gap showed that several researches that have been conducted have not been able to utilize multimedia data mining in monitoring e-examination environment. Also, the past security measure employed in the area of e-assessment was mostly based on single factor such as facial recognition, fingerprint recognition, e.t.c. which have been insufficient. So also, it can be deduced that in this domain, more consideration have been put in the aspect of authentication than in monitoring. This research proposes a framework therefore as a first step towards filling this gap.

\section{E-EXAMINATION ENVIRONMENT}

Today, many higher institutions of learning are creating virtual learning environment where learning can take place anytime, anywhere. Their aim was to support education with modern learning and teaching tools and to ensure that everyone have access to education. Therefore, the e-learning systems and emerging technologies suffer from vulnerability to different types of attacks such as unauthorized access to sensitive information, viruses, spyware, etc. They also lack effective mechanism for preventing and detecting cheating, impersonation and monitoring the eexamination environment to avoid illegal assistance.

In many e-learning environments, e-examination plays an important role in e-learning assessment and a critical asset in determining learner's performance. Previous research in e-learning assessment shows that student authentication in an e-examination setting face several security challenges [14]. Academic dishonesty has been a growing concern in e- learning environment due to the fact that e-examination takes place under supervised and unsupervised learning environment despite its huge advantages. For e-examination to take place student identification is very important and this relies on a secured authentication approach due to inherent anonymity of users on the e-learning platform. Also, the integrity of the examination and the examination environment is another important factor. The environment must be free from all forms of cheating especially in an unsupervised environment [15].

Online assessment is very important to ascertain students' progress [16]. Previous research has shown that $73.6 \%$ of students think that cheating in an online environment is easier than in traditional classroom setting [17]. Therefore, improving the security of E-learning and its environment will improve the security of online assessments, and this should not be neglected.

Some models have been proposed to secure the e-examination but did not take into consideration the environment in which the e-examination is taken place. Thus, the proposed system is an improvement on the existing models using combination of multimodal (fingerprint, face and voice recognition system) biometric authentication and multimedia data mining for extracting and analysing massive set of multimedia data (image, audio and video) in order to discover and obtain useful knowledge regarding all forms of academic dishonesty. This will help provide adequate security and reduce unethical behaviour in an e-examination environment. 


\section{RESEARCH METHODOLOGY}

This research work proposes a methodology that is based on the multimedia data mining processes. The environment here is a state whereby an examination candidate carries out a preexamination registration by providing the monitoring features (i.e. webcam for images and audio for voice recording) that will be utilized in the monitoring of the environment. The metadata is then extracted from these devices and stored in the multimedia database. The multimedia database contains unstructured data which is then converted to structured data and data mining tools are applied on the structured data. This procedure is based on the fact that every individual who engages in e-examination has these features.

\subsection{Proposed FrameWork}

The proposed framework will be employing the multimedia data mining techniques. The several phases of the multimedia data mining will be employed which comprises of the pre-processing, feature extraction, Data mining (i.e. classification, clustering, and association) and post processing. The proposed framework shown in Figure 1 will be explicitly explained in this section. It is a prototype representing how the entire system would work and operate.

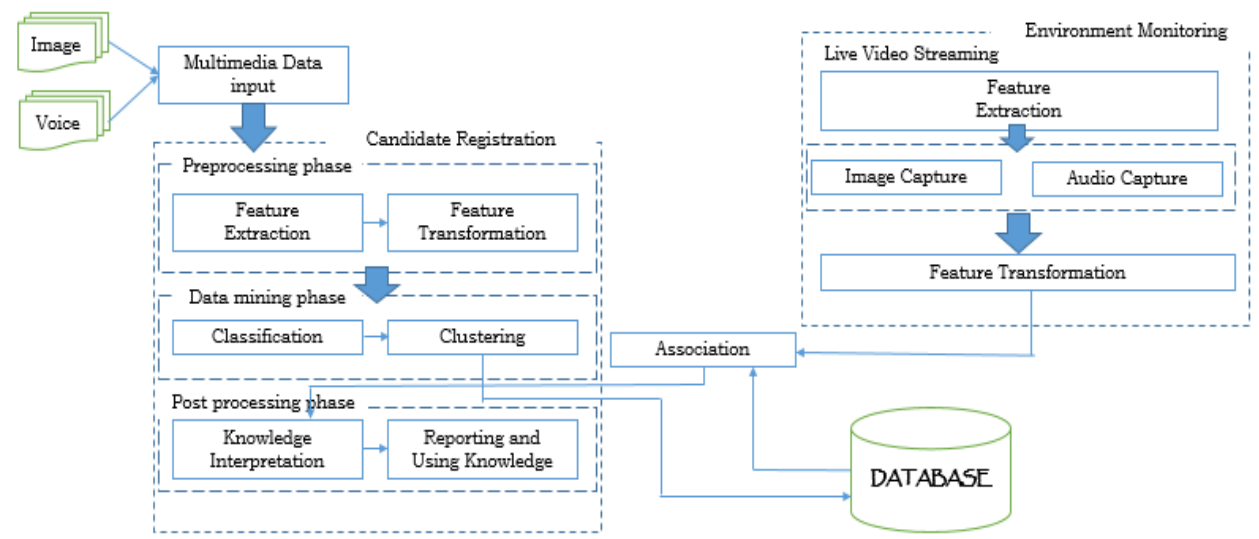

Figure 1: Proposed multimedia data mining framework for monitoring e-examination environment.

\subsubsection{MUlTimedia Data InPUT (MDI)}

Every data that must be mined must first be stored and made available in a storage before being mined. The two multimedia data type being considered in this work are the image and voice which will be collected as a raw data from participants who intend to take an e-examination. This data will be collected at registration so as to make use of it in monitoring the environment. The Multimedia Data Input (MDI) phase deals majorly with the capturing of image and voice of the participants which will be inculcated into the registration page of the e-examination platform. This process will require hardware devices that will get the capture of the faces and voices of the intending examination participants. The capture will therefore require the inclusion of drivers that will make the software work with the device.

\section{Algorithm for Multimedia Data Input}

INPUT: Frontal Image (FI), Voice Recording (VR)

OUTPUT: FI, VR 


\section{PROCESS:}

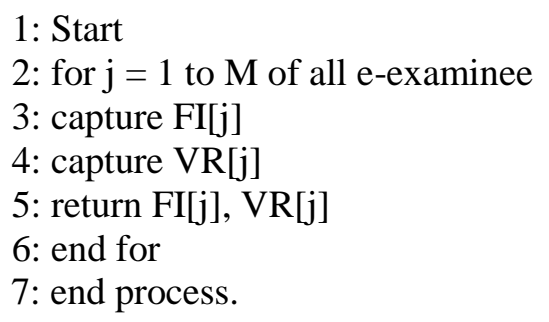

The algorithm from lines 2-6 shows that the operation will run for the number of times the set of multimedia data of all aspiring examinee captured during registaration. Assuming the number of inputs are $\mathrm{n}$, the algorithm has a time complexity of $\mathrm{O}(\mathrm{n})$.

\subsubsection{Pre-Processing Phase}

As a means to carry out proper verification of data which is being used in the system, it is necessary to preprocess the multimedia data. It is scientifically necessary since not every feature is considered in the verification, the needed features need to be identified which makes preprocessing necessary. These section is carried out after the registration of the candidate is completed. It comprises of two sub phases which are the features extraction and the features transformation.

\subsubsection{FEATURES EXTRACTION (FE)}

Feature extraction has to do with the reduction of resources necessary for the description of a large set of data, in this case the image, video and audio collected. Performing the analysis of complex data has a major problem which stems from the variables number involved. Analysing with a large number of variables requires generally large amount of memory and computation power which may lead to the over fitting of a classification algorithm for the training of samples. Feature extraction is a construction process which combines variables to get around these problems with data description that has sufficient accuracy.

\section{Algorithm for feature extraction}

INPUT: FI, VR

OUTPUT: Image feature (ImF), Voice feature (VoF).

PROCESS:

1: Start

2: for $\mathrm{i}=1$ to $\mathrm{N}$ of all examinee

3: Input FI[i], VR[i]

4: initialize $\mathrm{ShD}$

5: extract shape descriptor, $\mathrm{ShD}$ from $\mathrm{FI}[\mathrm{i}]$

6: $\mathrm{ImF}=\mathrm{ShD}$

7: initialize $\operatorname{ImgF}[]$

8: $\quad$ for $\mathrm{k}=1$ to $\mathrm{M}$

9: $\quad$ extract image features, $\operatorname{ImgF}[\mathrm{k}]$

10: end for

11: initialize FrS

12: extract frequency spectrum, FrS from VR

13: $\mathrm{VoF}=$ FrS

14: end for

15: return ImF, VoF

16: end process 
The algorithm from lines 2-5 shows that the operation will run for the number of aspiring examinee which gives it a time complexity of $\mathrm{O}(\mathrm{n})$ at that instance. For the presence of another loop from lines $8-10$, the time complexity is $\mathrm{O}\left(\mathrm{n}^{2}\right)$. This makes the entire algorithm have a time complexity of $\mathrm{O}\left(\mathrm{n}^{2}\right)$.

\subsubsection{FEATURES TRANSFORMATION (FT)}

Here, the extracted features are being transformed into a unit that will be storable in terms of a data type which will be storable so as to be used for matching and verification. Therefore, each feature that was given as input in FE will be tagged one after the other for a single examinee based on each individual feature. For example, if an examinee A has a voice and image collected, the image feature extracted could be labelled as Img_A_Featl, the audio feature as and Aud_A_Feat1. Therefore, the features have been transformed by renaming.

\subsubsection{Data Mining Phase}

This phase has to do with the classification of the multimedia data features that have been preprocessed and clustering them.

\subsubsection{Data Mining Classification (DMC)}

The transformed features is hereby classified as a group belonging to a class of file for a particular entity in this case, the examinee. Any of the features with same data file and name for a particular entity is classified as that file type for the entity. The video classification is done and collections of image features in it are kept in a class so as to be matched with any of same file for monitoring. The image feature of the examinee is also classified as an image file that will be used to check in future. This also applies to the audio file. The Bayesian classifier technique will be employed. It is a classification technique based on Bayes' Theorem with an assumption of independence among predictors. It assumes that the presence of a particular feature in a class is unrelated to the presence of any other feature.

\section{Algorithm for the Data Mining Classification}

INPUT: All multimedia features $\mathrm{MmF}$ ]

OUTPUT: Image_Class (Img_C), Audio_Class (Aud_C)

PROCESS:

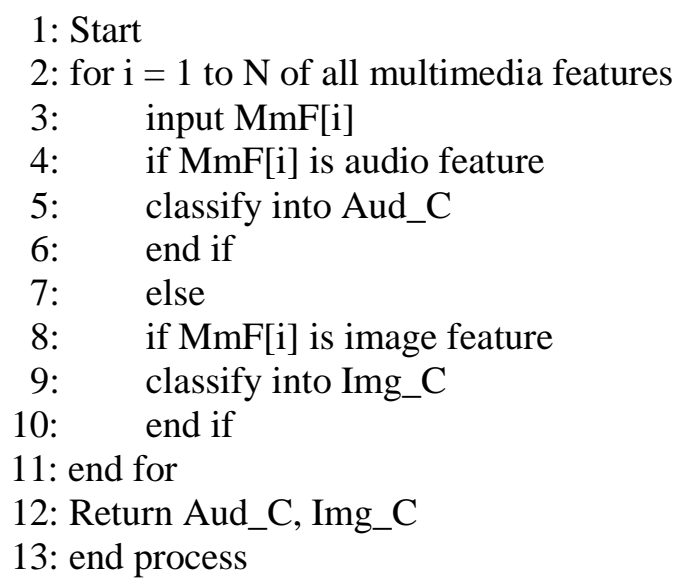

The algorithm from lines 2-11 shows that the operation will run for the number of multimedia data features in the database. Each of the conditional section in lines 4-6, 7-10 have a time 
complexity of $\mathrm{O}(1)$. Assuming the number of inputs are $\mathrm{n}$, the algorithm has a time complexity of $\mathrm{O}(\mathrm{n})$ which is the outer loop which controls the algorithm.

\subsubsection{Data Mining Clustering (DMCL)}

The data mining clustering creates a branch of the classified information which represents multimedia belonging to a particular entity as one cluster so as to identify the examinee if he/she is present in an examination environment. The root description serves as the label for any feature that is found to be similar with the monitoring data. These information are then being stored in the system database for future use.

\section{Algorithm for DMCl}

INPUT: All multimedia data features

OUTPUT: cluster roots (clust[])

PROCESS:

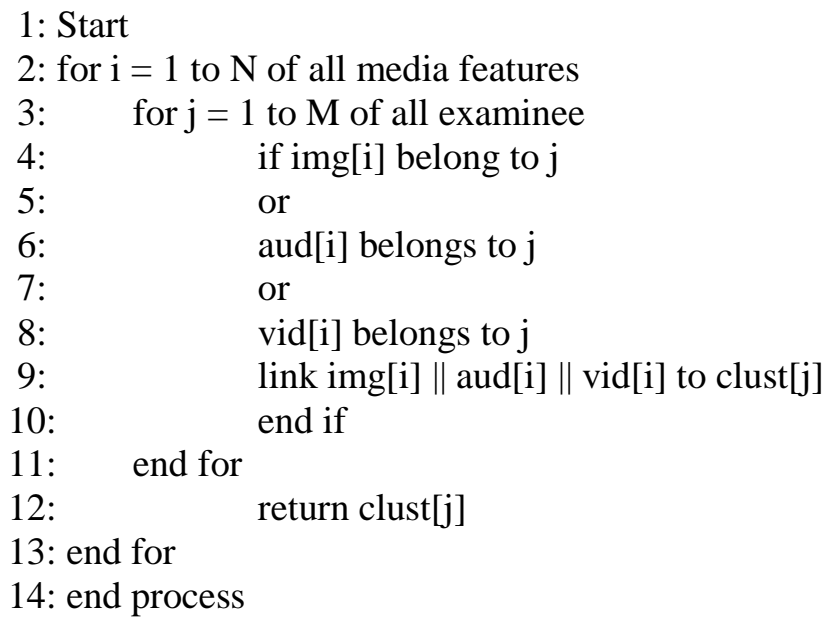

The algorithm from lines 2-13 shows that the operation will run for the number of media features which gives it a time complexity of $\mathrm{O}(\mathrm{n})$ at that instance. For the presence of another loop from lines 3-11, the time complexity is $\mathrm{O}\left(\mathrm{n}^{2}\right)$. This makes the entire algorithm have a time complexity of $\mathrm{O}\left(\mathrm{n}^{2}\right)$.

\subsubsection{EXAMINATION ENVIRONMENT MONITORING}

This phase of the work comprises of the video streaming phase and feature transformation. Videos are media that are made up of images and audio sounds. The monitoring camera get time to time instance of captured images from a video shot in an e-examination environment as well as audio sound from a central microphone that will be utilized. The feature extraction and transformation technique employed in section 4.1.2 is also being adopted here so as to use it for matching the pattern of the stored features.

\subsubsection{DATA Mining ASSOCIATION (DMA)}

This phase carries out the matching of data features in an e-examination environment. Each media data features that are of the same type are compared with the stored features to determine if the image captured at monitoring is present in the database or voice captured is present with any in the system database. 


\section{Algorithm for DMA}

INPUT: monitoring image features (m_img), monitoring audio (m_aud), all database features (db_feat[])

OUTPUT: cluster root (clust) OR invalid response

PROCESS:

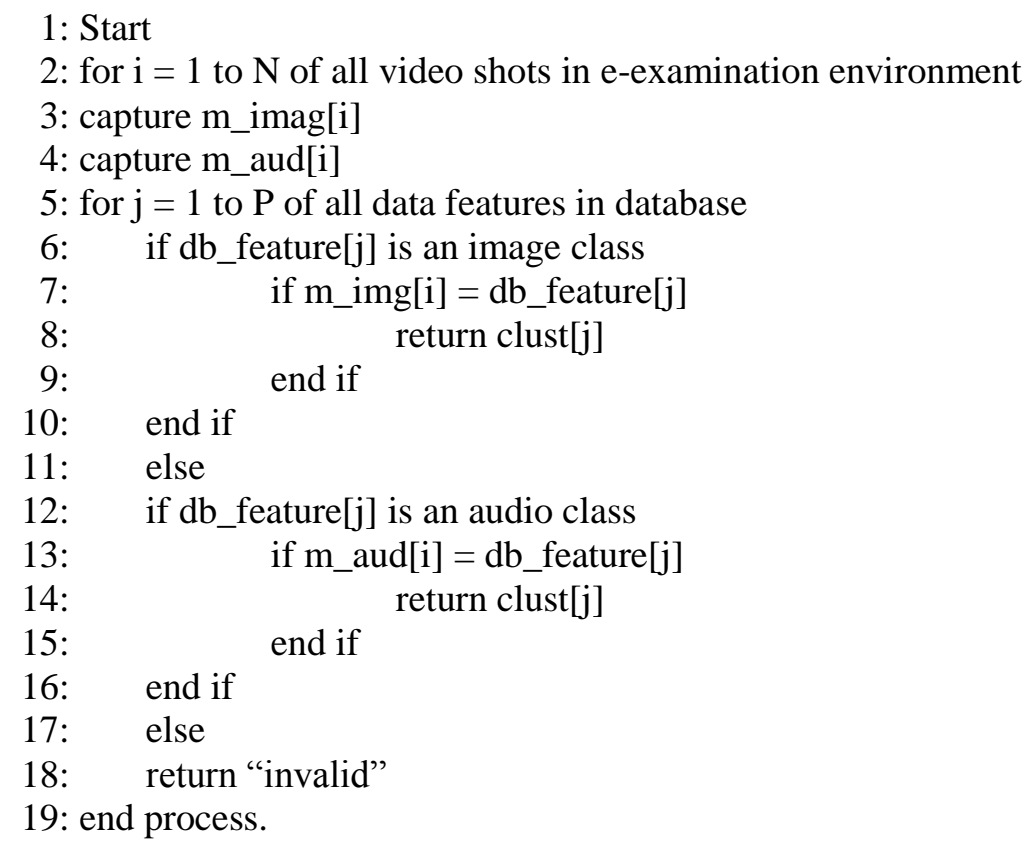

The algorithm from lines 2-20 shows that the operation will run for the number of video shots which gives it a time complexity of $\mathrm{O}(\mathrm{n})$ at that instance. For the presence of another loop from lines 5-19, the time complexity is $\mathrm{O}\left(\mathrm{n}^{2}\right)$. Each of the conditional statements in lines 6-10, 7-9, 12$16,13-14$ are each having a time complexity of $\mathrm{O}(1)$. This makes the entire algorithm have a time complexity of $\mathrm{O}\left(\mathrm{n}^{2}\right)$.

\subsubsection{Post Processing}

The post processing phase is centered on displaying the necessary output that the data mining association phase have carried out in human understandable form. As the cluster cannot be clear for the user to comprehend, so the knowledge need to be interpreted and reported for user's understanding. The feature that was transformed is hereby reversed back to the original label while in the reporting phase, the name of the candidate is being represented if his/her feature is present. If such feature is not present in the database, the system gives a notification report that an unregistered individual is present in the examination environment. This will hereby call for the action of the examination coordinators concerning the unauthorized individual.

\section{CONCLUSION}

The study presented a framework for monitoring e-examination environment using multimedia data mining which can be employed in detecting the presence of an unauthorized candidate in an e-examination arena. It was discovered from research that this approach has not been employed in the monitoring of e-examination activities which have led to various breeches of rules. The monitoring is based on the employing of image and audio files in the monitoring of the environment. The image if not sufficient is being complimented by the audio input. The idea placed in this study is rather generic and at the early stage. This therefore necessitates for further 
research in the implementation of this proposal in a real environment which will make the evaluation of this work to be done. Beyond employing this framework in an e-assessment environment, it can also be used in monitoring work environments such as the banking industry.

\section{ACKNOWLEDGEMENTS}

We like to acknowledge and appreciate all lecturers and Postgraduate students of Computer Science Department, Babcock University for their moral support and encouragement during this research. Thank you and God bless.

\section{REFERENCES}

[1] Amie, R and McKibban, C.A.B. (2013). Academic Dishonesty: An In-Depth Investigation of Assessing Measurable Constructs and a Call for Consistency in Scholarship. Journal of Academic Ethics, 11(3): pp. 185-197.

[2] King, C., Guyette, R., \& Piotrowski, C. (2009). Online exams and cheating: An empirical analysis of business students 'views. The Journal of Educators Online, 6(1), pp. 1-11.

[3] Watson, G. and Sottile, J. (2010). "Cheating in the Digital Age: Do Students Cheat More in Online Courses”. Online Journal of Distance Learning Administration, 13(1), ISSN pp. 1556-3847.

[4] Jun-Ming, S., Shian-Shyong, T., Wei, W., Jui-Feng, W., Jin-Tan, D. and Wen-Nung, T. (2006).

[5] Watters, M. P., Robertson, P. J. and Clarke, R. K. (2011). "Student perceptions of cheating in online business courses". Journal of Instructional Pedagogies, pp 1-7

[6] Northcutt, C. G., Ho, A. D., and Chuang, I. L. (2015) "Detecting and preventing multiple-account cheating in massive open online courses,"arXiv preprint arXiv: 1508.05699.

[7] Manjunath, R. and Balaji, S. (2014). "Review and Analysis of Multimedia Data Mining Tasks and Models," IJIRCCE, Bengaluru, India, vol. 2, pp. 124-130.

[8] Vijayarani, S., \& Sakila, A. (2015). Multimedia Mining Research-An Overview. International Journal of Computer Graphics \& Animation, 5(1), pp. 69.

[9] Amigud, A., Arnedo-Moreno, J., Daradoumis, T., \& Guerrero-Roldan, A. E. (2016). A Behavioral Biometrics Based and Machine Learning Aided Framework for Academic Integrity in E-Assessment. In Intelligent Networking and Collaborative Systems (INCoS), 2016 International Conference on (pp. 255-262). IEEE.

[10] Pesare, E., Roselli, T., Rossano, V., \& Di Bitonto, P. (2015). Digitally enhanced assessment in virtual learning environments. Journal of Visual Languages \& Computing, 31, 252-259.

[11] Dhakar, M., \& Tiwari, A. (2014). A novel data mining based hybrid intrusion detection framework. Journal of Information and Computing Science, 9(1), pp. 037-048.

[12] Banda, J. M., Angryk, R. A., \& Martens, P. C. (2013). Imagefarmer: Introducing a data mining ramework for the creation of large-scale content-based image retrieval systems. International Journal of Computer Applications, vol. 13 pp. 79.

[13] Chen S., Shyu, S., Zhang C., Strickrott J. (2002). Multimedia Data Mining for Traffic Video Sequences. Journal of Intelligent Information Systems. Vol. 19(1), pp 61-77

[14] Gathuri, J. W., Luvanda, A. Matende, S. and Kamundi, S. (2014). Impersonation Challenges Associated With E-Assessment of University Students. Journal of Information Engineering and Applications www.iiste.org ISSN 2224-5782 (print) ISSN 2225-0506 (online), Vol. 4(7).

[15] Northcutt, C.G., Ho, A.D., \& Chuang, I. L. (2015). Detecting and preventing "multiple-account" cheating in Massive Open Online Courses. arXiv:1508.05699 [cs.CY].

[16] Meyer, J.P., \& Zhu, S. (2013). Fair and equitable measurement of student learning in MOOCs: An introduction to item response theory, scale linking, and score equating. Research \& Practice in Assessment, Vol. 8(1), pp. 26-39.

[17] Guyette, R. W. and Piotrowski, C. (2009). Online exams and cheating: An empirical analysis of business students'views, The Journal of Educators Online, Vol. 6(1), pp. 1-11. 
The International Journal of Multimedia \& Its Applications (IJMA) Vol.9, No.3, June 2017

\section{Authors}

Mrs. B. T. Adetoba is a Postgraduate student at Babcock University, Ilishan, Ogun State, Nigeria. She has Bachelor of Science and Master of Science in Computer Science from Obafemi Awolowo University and University of Lagos, Nigeria, respectively. She is a member of Computer Professionals of Nigeria (CPN) and Nigeria Computer Society (NCS).

Prof. O. Awodele is the Dean of School, Babcock University, Ilishan-Remo, Ogun State; Nigeria. He holds a Ph.D. in Computer Science and his research Interests are in the area of Artificial Intelligence and E-learning. He is a fellow of Nigeria Computer Society (NCS) and Computer Professionals of Nigeria (CPN).

Dr. Kuyoro, S. O. is a Senior Lecturer in Computer Science Department, Babcock University, Ilishan-Remo, Ogun State; Nigeria. She holds a Ph.D. in Computer Science and her research interest is in machine learning. She is a member of Nigeria Computer Society (NCS) and Computer Professionals of Nigeria (CPN). 